\title{
Comorbid physical illnesses in adult outpatients with psychotic disorders: risk factors, psychological functioning, and quality of life outcomes
}

\author{
Wen Lin Teh ${ }^{1}$ (D) Laxman Cetty $^{1} \cdot$ Anitha Jeyagurunathan ${ }^{1} \cdot$ Fiona Devi $^{1} \cdot$ Kumarasan Roystonn $^{1} \cdot$ Charmaine Tang $^{2}$. \\ Swapna Verma ${ }^{2} \cdot$ Mythily Subramaniam ${ }^{1}$
}

Received: 14 October 2020 / Accepted: 29 January 2021 / Published online: 22 February 2021

(C) The Author(s) 2021

\begin{abstract}
Purpose In contrast to global research, where physical comorbidity in psychotic disorders is established, only a few studies have been conducted in Southeast Asia. With a concerning trend of chronic physical illnesses emerging in adults below the age of 65, an investigation into comorbid chronic physical illnesses in adults diagnosed with psychotic disorders is necessary. This study aims to explore the risk factors, psychological functioning, and quality of life outcomes associated with comorbidity in adults below the age of 65, diagnosed with psychotic disorders, in a multi-ethnic non-Western setting.

Methods Electronic medical records of 364 patients with psychotic disorders who had provided written consent to participate were screened for co-occurring physical conditions. The majority of participants were female (53.7\%), Chinese (69\%), single (74.5\%), and had tertiary and above education (43\%). They were approximately 35 years old on average and the mean age of onset for psychosis was 26.7 years old.

Results Comorbid physical illnesses were present in approximately a third of adults with psychotic disorders (28\%). They typically reported cardiovascular-related diseases, respiratory, and skin conditions. Comorbidity was significantly related to lower physical quality of life. As compared to other types of psychotic disorders, schizophrenia was significantly related to a greater frequency of comorbid physical conditions. Multinomial regression analyses revealed that age, age of onset, Malay and Indian ethnicities were significant factors.

Conclusion Physical comorbidity in adults below the age of 65 is common, signifying an emerging need to place greater attention into the screening and emphasis on the physical care needs of this age group. Finally, more research is needed to understand the impact of common co-occurring acute and chronic cardiovascular, skin, and respiratory diseases locally.
\end{abstract}

Keywords Physical comorbidity · Psychotic disorders · Quality of life · Psychological health

\section{Introduction}

When compared to the general population, individuals with psychotic disorders die 10-15 years prematurely on average $[1,2]$. A large proportion of premature mortality is attributed to comorbid medical conditions [1-6] which are common

Wen Lin Teh

Wen_Lin_Teh@imh.com.sg

1 Research Division, Institute of Mental Health, Buangkok Green Medical Park, 10 Buangkok View, Singapore 539747, Singapore

2 Department of Early Psychosis Intervention, Institute of Mental Health, Singapore 539747, Singapore in psychotic disorders. Adapted from Akker et al. [7] and originally from Feinstein et al. [8], comorbidity is defined as any co-occurring chronic condition in addition to the index disease. Depending on the type of medical conditions, physical comorbidity may occur in up to $75 \%$ of individuals with psychotic disorders $[9,10]$. They are more likely to report diabetes, neurological disorders, metabolic conditions, and respiratory illnesses than the general population [3, 9-14]. Comorbidity is influenced by sociodemographic factors, such as age [15] and gender [9, 16]. While chronic conditions are commonly studied in older adult populations (defined typically in epidemiological studies as $\geq 65$ years old) as it is conventionally assumed to be conditions of old age, research has shown that chronic diseases, such as diabetes and cardiovascular diseases, are becoming increasingly 
common in adults below the age of 65 years [17], and is a concerning trend among adults with severe mental illnesses [18].

Cardiovascular diseases, diabetes, chronic respiratory diseases, and cancer are the four chronic noncommunicable diseases with the highest cause of death and disability in Southeast Asia which is similar to global trends [19]. However, Asian populations have increased morbidity and mortality in important chronic conditions, such as coronary heart disease, as compared to Western populations [20]. Singapore is a Southeast Asian country with approximately four million residents who belong to three major ethnic groups (Chinese, Malay, and Indian). Epidemiological surveys conducted in Singapore found that ethnic minorities in Singapore generally self-report greater physical chronic illnesses [21-23] and while the prevalence of severe mental illnesses in the major ethnic groups are established [24], little is known about physical medical conditions in relation to ethnicity and severe mental illnesses, particularly in psychotic disorders.

In contrast to global research, where physical comorbidity in psychotic disorders is established, only a few studies have been conducted in Southeast Asia. It remains unclear the extent to which global trends concerning comorbid presentations in psychotic disorders are generalizable to and descriptive of a non-western population that is also multi-ethnic. Epidemiological surveys that investigate severe mental illnesses typically do not include questions on psychotic disorders in shorter questionnaires due to the lack of validity of self-report in diagnosing psychotic disorders [3, 25, 26], contributing to the lack of data. Existing local studies that use administrative data are however limited to the study of schizophrenia only [27, 28], whereas other schizophrenia spectrum disorders and mood disorders with psychotic symptoms are largely ignored. Consequently, local research investigating the impact of physical comorbidity on psychosocial outcomes, such as quality of life (QOL) and psychological functioning, among individuals with psychotic disorders is generally lacking. Lastly, while it is typical for studies to include chronic medical conditions since they are more pervasive in older adults [7,8], such strict inclusion criteria has inevitably contributed to a lack of breadth in terms of the scope of medical conditions investigated. Acute medical conditions, such as bacterial infections (i.e., tuberculosis) remain poorly studied as compared to chronic conditions like diabetes mellitus [12].

Given the present gaps in the current literature, this study aims to explore the types of comorbid physical illnesses, risk factors, psychological and quality of life outcomes of comorbid physical disorders in younger adult patients with psychotic disorders. From past reports on various severe mental illnesses, it is hypothesized that having comorbid medical conditions would be significantly associated with older age and lower quality of life [15,
$26,29,30]$. Supplementary to the study of comorbidity, we aim to also investigate co-occurring acute and chronic medical conditions at a descriptive level and its relation to QOL and psychosocial outcomes, as there is an exploratory value to do so [7].

\section{Methods}

\section{Procedure}

Outpatients with psychotic disorders were recruited from the Institute of Mental Health (IMH), a tertiary psychiatric hospital in Singapore, between January 2018 and April 2019. According to the Diagnostic and Statistical Manual for Mental Disorders (DSM-IV), psychotic disorders refer to a group of illnesses that are characterized by hallucinations, delusions, disorganized speech, grossly disorganized behavior, and negative symptoms [31]. Participants were referred to the research team by their clinicians, who were aware of the full objectives of the research study, or they had approached the research team in the outpatient clinics. Participants were recruited using an information flyer that described the details of the study. Participants' diagnoses were confirmed using their electronic medical records or by their doctors. Participants who agreed to participate signed written informed consent forms prior to completing a set of questionnaires by paper and pen. Their responses were subsequently entered electronically by the research team to prepare for analyses. The inclusion criteria for the study were: Singapore citizens or Permanent residents, aged 21-65 years old, have a history of a psychotic disorder, and are literate in the English language. The exclusion criteria were substanceinduced psychotic disorders (as they have distinct clinical and demographic presentations than primary psychotic disorders [32]), those with intellectual disabilities, and those who were unable to read or speak in English. This study was approved by the relevant institutional review committees-the National Health Group (NHG) Domain Specific Review Board (DSRB) and the IMH Institutional Research Review Committee (IRRC).

\section{Materials}

Medical records of patients who provided written consent to participate in this study were screened. For the purposes of this study, we extracted the primary psychiatric diagnoses, existing acute and chronic medical conditions, and age of onset of psychotic disorders. Participants self-reported their age, marital status, gender, highest education levels, and ethnicity. 


\section{Measures}

i. Depression Anxiety Stress Scales (DASS-21) [33]: This is the short version of DASS that measures subscales of Depression, Anxiety, and Stress (seven items per subscale). The DASS-21 scale asks participants to respond the extent to which the statements applied to them over the past week. Likert responses range from 0 (never) to 3 (almost always). This is a valid measure for use on clinical and non-clinical samples [34]. Internal reliability of the subscales in the present sample was excellent, ranging from 0.83 to 0.90 .

ii. World Health Organization-Quality of Life (WHOQOL-BREF) [35]: This questionnaire measures four domains of quality of life: physical health, psychological health, social relationships, and environment. This scale had been found to a valid measure for QOL in psychiatric outpatients including psychotic disorders [36]. Cronbach alpha ranged from acceptable ( 0.6 for the physical component) to excellent ( 0.8 for the environment component) in this study.

\section{Physical medical conditions}

As a large number of medical conditions were evaluated, we grouped individual (chronic and acute) conditions into 15 categories that represented the 10th version of the International Statistical Classification of Diseases and Related Health Problems (ICD-10). Classifying individual medical conditions into ICD-10 medical categories ensured adequate parsimony and sufficient statistical power for analyses involving rarer medical conditions [37]. The 15 categories were: neoplasm (e.g., mixed glioma, carcinoma, etc.), infectious or parasitic diseases (e.g., tuberculosis, viral warts, varicella, etc.), disorders of the circulatory system (e.g., myocardial infarction, stroke, etc.), skin (e.g., dermatitis, alopecia, etc.), musculoskeletal system and connective tissue (e.g., scoliosis, arthropathy, back pain, etc.), digestive (e.g., enteritis, irritable bowel syndrome, etc.), respiratory (e.g., asthma, rhinitis, etc.), ear/mastoid (e.g., tinnitus, otitis media, etc.), adnexa/eye (e.g., conjunctivitis, myopia, etc.), nervous system (e.g., epilepsy, migraine, tardive dyskinesia, etc.), blood (e.g., anemia, etc.), endocrine/metabolism/nutritional (e.g., hyperlipidemia, obesity, diabetes mellitus, etc.) and genitourinary tract (e.g., bladder stone, amenorrhea, etc.).

To study comorbidity, a subset of these conditions were subsequently re-categorized into nine categories representing fifteen chronic conditions from the composite international diagnostic interview (CIDI) checklist [26]. These categories were typically used in local population-based epidemiological studies as they were locally relevant and selected based on the advice by various stakeholders [38]. They were: respiratory conditions (asthma, emphysema), diabetes or hyperglycemia, hypertension, hyperlipidemia, chronic pain (arthritis, rheumatism, migraine, back problems), cancer, neurological disorders (epilepsy, Parkinson's disease), cardiovascular diseases (stroke, heart attack, angina, or other heart diseases), ulcer and chronic inflamed bowel (stomach ulcer, enteritis, colitis). Comorbidity was operationalized as having at least one comorbid chronic condition. A comorbid condition thus refers to a co-occurring chronic medical/physical condition in addition to the index condition of psychotic disorders.

\section{Statistical analyses}

The initial calculation of descriptive statistics, such as the means and standard deviations, were conducted to understand the distribution of the sample's characteristics. Analysis of Variance (ANOVA) and test of independence chi-square tests were conducted to ascertain if there were significant differences/associations between the three groups and sociodemographic variables. Phi coefficients were calculated to determine the correlation between physical categories which had dichotomous indicators (Yes/No). Non-parametric Kruskal-Wallis tests were utilized to determine if there were significance differences in the frequency of general medical and comorbid conditions between three psychotic disorder groups. To investigate the risk factors of comorbidity, multinomial logistic regression was conducted using three outcomes: none, one, and two or more comorbid conditions. Finally, multiple linear regression analyses were conducted to ascertain if there were significant associations between comorbidity and anxiety, depression, or stress levels (as measured by DASS-21), and four dimensions of quality of life such as physical health, psychological health, social relationships, and environment QOL (as measured by WHOQOL-BREF), while accounting for sociodemographic covariates.

To explore the impact of co-occurring ICD-10 general medical conditions (pertaining to all physical conditions regardless of chronicity) in individuals with psychotic disorders, separate descriptive statistics, ANOVA, the calculation of phi coefficients, and multiple linear regression analyses were conducted. All tests utilized a 0.05 criterion for statistical significance and were conducted in IBM SPSS Statistics for Windows, version 23. The results are presented as supplementary material of this manuscript. Missing values were deleted listwise. 


\section{Results}

\section{Sample characteristics}

Data of 364 participants with psychotic disorders were utilized for this study. Participants were approximately 35 years old on average (ranging from 21 to 65 years old), and the mean age of onset was 26.7 years. One participant was missing a data point on diagnosis, and hence the analyses involving comparison between psychotic disorder groups were based on 363 participants. To be consistent with the classification of psychotic disorders in the Diagnostic and Statistical Manual of Mental Disorders (DSM-5), individuals with schizophrenia, schizoaffective disorder, schizophreniform disorder, brief psychotic disorder, delusional disorder, and psychotic disorder NOS were initially classified as non-affective psychotic disorders. However, as preliminary analyses found that the other non-affective psychotic disorder group (age: $M=32.3$, $S D=9.5$ ) was significantly younger than those with schizophrenia (age: $M=37.2, S D=11$ ), and as initial chi-square analyses suggested that individuals with schizophrenia had greater comorbid conditions, it was decided to separate individuals with schizophrenia from the rest of the nonaffective psychotic disorder as a separate group. Individuals with either Bipolar I disorder or Major Depressive Disorder and with psychotic features were classified as having affective psychotic disorders (age: $M=27, S D=7.6$ ). Age of onset was not significantly different between the three psychotic disorder categories. Majority of participants were female (53.7\%), Chinese (69\%), single (74.5\%), and had tertiary and above education (43\%). There were significant differences in only one domain of QOL (social relationships) between the psychotic disorder groups. Post hoc pair-wise comparisons using Bonferroni corrections revealed a marginal significant difference between schizophrenia and other non-affective psychotic disorder groups $(p=0.05)$. The results are summarized in Table 1.

\section{Comorbidity between groups}

Almost a third (28\%) of participants with psychotic disorders had at least one comorbid condition. $17 \%$ of participants had one comorbid condition only whereas $11 \%$ of participants had two or more comorbid conditions. Hyperlipidemia was the most prevalent comorbid condition and approximately $14 \%$ of participants with psychotic disorders had the condition. This was followed by diabetes or hyperglycemia $(7.4 \%)$, chronic pain $(7.1 \%)$, respiratory conditions $(6.1 \%)$, and others. When considering all general medical conditions (i.e., regardless of chronicity), approximately half (49.3\%) of all participants had at least one condition. Majority had respiratory or skin conditions (25.5\%).

As the statistical distribution of the frequency count of comorbid conditions appeared positively skewed (i.e., most participants had none as compared to six comorbid conditions), we performed non-parametric Kruskal-Wallis tests to determine if there were significant differences in the frequency count of comorbid conditions between the three psychotic disorder groups. Significant differences were present, $\chi^{2}(2)=9.72, p=0.008$, among the three groups. Post hoc pairwise comparisons using Kruskal-Wallis tests revealed that there were significant differences between schizophrenia and non-affective psychotic disorder groups, $\chi^{2}(1)=6.63$, $p=0.01$, and between schizophrenia and affective psychotic disorder groups, $\chi^{2}(1)=4.07, p=0.044$. There were no significant differences between other non-affective and affective psychotic disorder groups. These results are summarized in Table 2. No significant differences were recorded for general medical conditions (supplementary Table 1).

\section{Associations of comorbidity}

Multinomial logistic regression revealed that older age, earlier age of onset, Malay and Indian ethnic groups (as compared to Chinese ethnic group) were risk factors associated with greater odds of having one, and two or more comorbid conditions as compared to none. Marital status, education levels, and the type of psychotic diagnosis were not significantly associated with comorbidity (Table 3).

Multivariate linear regression analyses revealed that comorbidity was not associated with the majority of psychological and QOL (except for physical health QOL) domains after accounting for sociodemographic information, psychotic disorder type, and age of onset of psychosis. Having one comorbid condition (as compared to none) was significantly and negatively associated with physical health and social relationships QOL scores (Table 4). Having at least one co-occurring medical condition (as compared to none) was associated with higher levels of anxiety, depression, stress DASS-21 scores, and lower physical health QOL scores. Having at least two medical conditions (as compared to none), was significantly associated with lower physical health QOL scores only (supplementary Table 2).

Results on co-occurring general medical conditions are summarized in the supplementary section.

\section{Discussion}

Thirty percent of individuals with psychotic disorders had at least one comorbid physical condition. These results were lower than those reported elsewhere among individuals with 
Table 1 Sample characteristics, DASS-21, and WHOQOL-BREF scores among psychotic disorder groups

\begin{tabular}{|c|c|c|c|c|c|}
\hline & $\begin{array}{l}\text { All } \\
(n=364)\end{array}$ & $\begin{array}{l}\text { Schizophrenia } \\
(n=231)\end{array}$ & $\begin{array}{l}\text { Other non-affective psy- } \\
\text { chotic disorders } \\
(n=109)\end{array}$ & $\begin{array}{l}\text { Affective psychotic } \\
\text { disorders } \\
(n=23)\end{array}$ & \\
\hline Mean (sd) & & & & & $F\left(d f^{b}, d f^{v}\right)$ \\
\hline Age & $35.2(10.8)$ & $37.2(11)$ & $32.3(9.5)$ & $27(7.6)$ & $16.0(2,357)$ \\
\hline Age of onset & $26.7(7.93)$ & $26.8(7.8)$ & $26.9(8.5)$ & $23.9(4.2)$ & $1.48(2,356)$ \\
\hline \multicolumn{6}{|l|}{ DASS-21 scores } \\
\hline Anxiety & $10.2(8.8)$ & $10.1(8.7)$ & $9.9(8.7)$ & $12(10.6)$ & $0.53(2,358)$ \\
\hline Depression & $11.1(10.3)$ & $11.4(10)$ & $10(10.1)$ & $14.4(12.7)$ & $1.95(2,358)$ \\
\hline Stress & $11.4(10)$ & $11.7(10)$ & $10.2(9.5)$ & $14.7(11.3)$ & $2.18(2,357)$ \\
\hline \multicolumn{6}{|l|}{ WHOQOL-BREF scores } \\
\hline Physical health & $14.3(2.5)$ & $14.1(2.6)$ & $14.5(2.5)$ & $14.6(2)$ & $1.06(2,357)$ \\
\hline Psychological health & $12.9(3.1)$ & $12.8(3.2)$ & $13.3(2.8)$ & $12.4(2.9)$ & $1.17(2,359)$ \\
\hline Social relationships & $13.2(3.2)$ & $12.9(3.3)$ & $13.8(2.7)$ & $12.4(3.2)$ & $3.41(2,327)$ \\
\hline Environment & $13.7(2.8)$ & $13.5(2.9)$ & $14(2.4)$ & $14.3(2.6)$ & $1.98(2,358)$ \\
\hline$n(\%)$ & & & & & $\chi^{2}$ \\
\hline Gender & & & & & $n s$ \\
\hline Female & $196(53.7)$ & $124(34.2)$ & $55(15.2)$ & $16(4.4)$ & \\
\hline Male & $168(46.0)$ & $107(29.5)$ & $54(14.9)$ & $7(1.9)$ & \\
\hline Ethnicity & & & & & $n s$ \\
\hline Chinese & $252(69.0)$ & $157(43.3)$ & $76(20.9)$ & $19(5.2)$ & \\
\hline Malay & $57(15.6)$ & $39(10.7)$ & $15(4.1)$ & $2(0.6)$ & \\
\hline Indian & $40(11.0)$ & $26(7.2)$ & $12(3.3)$ & $2(0.6)$ & \\
\hline Others & $15(4.1)$ & $9(2.5)$ & $6(1.7)$ & $0(0)$ & \\
\hline Marital status & & & & & $n s$ \\
\hline Single & $272(74.5)$ & $176(48.5)$ & $82(22.6)$ & $14(3.9)$ & \\
\hline In a relationship/Married & $71(19.5)$ & $38(10.5)$ & $24(6.6)$ & $8(2.2)$ & \\
\hline Divorced/separated/ widowed & $21(5.8)$ & $17(4.7)$ & $3(0.8)$ & $1(0.3)$ & \\
\hline Highest education & & & & & 9.8 \\
\hline Secondary and below & $102(27.9)$ & $73(20.1)$ & $26(7.2)$ & $2(0.6)$ & \\
\hline Pre-tertiary & $105(28.8)$ & $112(30.9)$ & $47(12.9)$ & $11(3)$ & \\
\hline Tertiary and above & $157(43.0)$ & $46(12.7)$ & $36(9.9)$ & $10(2.8)$ & \\
\hline
\end{tabular}

$F$ and $\chi 2$ (Chi-square) statistics are based on Analysis of Variance (ANOVA) and test of independence chi-square tests; values in bold indicate significance at $p<.05 ; n s$ indicate not significant. $d f^{b}, d f^{\mathrm{w}}$ refer to between and within degrees of freedom, respectively

psychotic disorders or with other forms of severe mental illnesses $[9,13,16,26]$, with an exception of one study which found comorbidity in approximately $22 \%$ of outpatients with schizophrenia which was lower than the present figures [27]. Given the influence of age on comorbidity, the general lack of agreement between past and present findings were expected since the present sample population consisted of younger adults (i.e., the average age of the current sample was 35 years old). Furthermore, the lack of agreement could be explained by differences in methodology (i.e., the use of either self-report vs. medical records).

Regardless of the disparity in the proportions however, the trend of comorbidity remained largely the same for all non-affective psychotic disorders. Certain comorbid conditions, such as chronic pain, hypertension, diabetes, hyperlipidemia, and respiratory conditions were more common, which corroborated past findings [9, 16, 39]. These results may be explained by a multitude of underlying factors, such as genetics, lifestyle risk factors, and physiopathological vulnerabilities that are expressed in those presenting with psychotic disorders [40-44]. Unhealthy lifestyle habits, such as smoking, poor diet, and sedentary behaviors too may contribute to the comorbid presentations of cardiovascular and metabolic-related disorders [45, 46]. Comparisons between the three psychotic disorder groups revealed that individuals with schizophrenia had significantly greater frequency of comorbid conditions. This was expected as schizophrenia, relative to other non-affective or affective psychotic disorders, is more debilitating, such as having more pronounced (negative) symptoms [47], which 


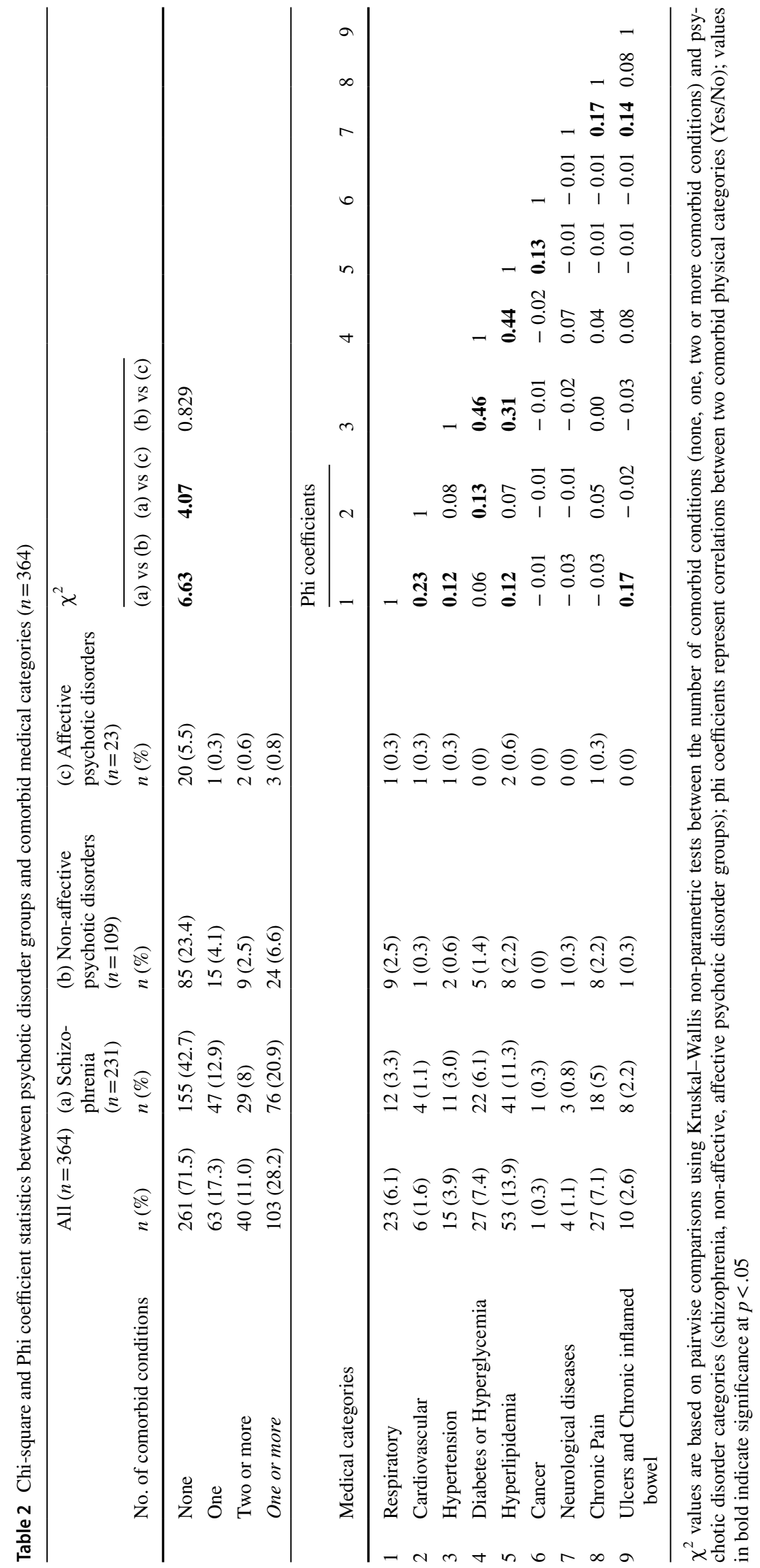


Table 3 Risk factors of comorbidity

\begin{tabular}{|c|c|c|c|c|c|c|c|c|c|}
\hline & \multirow{3}{*}{$\begin{array}{l}\text { None }^{\wedge} \\
n(\%)\end{array}$} & \multirow{3}{*}{$\begin{array}{l}\text { One }^{\wedge} \\
n(\%)\end{array}$} & \multirow{3}{*}{$\begin{array}{l}\text { Two or more } \\
n(\%)\end{array}$} & \multicolumn{6}{|c|}{ Multinomial Regression Analyses } \\
\hline & & & & \multicolumn{3}{|c|}{ One vs None } & \multicolumn{3}{|c|}{ Two vs None } \\
\hline & & & & OR & $95 \% \mathrm{CI}$ & $P$ value & OR & $95 \% \mathrm{CI}$ & $P$ value \\
\hline Age (Mean (SE)) & $33.2(0.6)$ & $37.8(1.5)$ & $42.9(1.8)$ & 1.08 & $1.042,1.121$ & $<.001$ & 1.12 & $1.076,1.168$ & $<.001$ \\
\hline Age of Onset (Mean (SE)) & $26.5(0.5)$ & $26.5(1.1)$ & $27.7(1.4)$ & 0.94 & $.898,0.987$ & 0.013 & 0.94 & $.891,0.989$ & 0.018 \\
\hline \multicolumn{10}{|l|}{ Sex } \\
\hline Female & $146(40.1)$ & $32(8.8)$ & $18(4.9)$ & 0.78 & $0.427,1.445$ & 0.437 & 0.61 & $0.285,1.305$ & 0.202 \\
\hline Male & $115(31.6)$ & $31(8.5)$ & $22(6)$ & 1 & & & & & \\
\hline \multicolumn{10}{|l|}{ Ethnicity } \\
\hline Chinese & $192(52.7)$ & $37(10.2)$ & $23(6.3)$ & 1 & & & & & \\
\hline Malay & $34(9.3)$ & $14(3.8)$ & $9(2.5)$ & 3.55 & $1.568,8.048$ & 0.002 & 3.41 & $1.248,9.336$ & 0.017 \\
\hline Indian & $22(6)$ & $11(3)$ & $7(1.9)$ & 3.45 & $1.462,8.150$ & 0.005 & 3.32 & $1.134,9.701$ & 0.029 \\
\hline Others & $13(3.6)$ & $1(0.3)$ & $1(0.3)$ & 0.50 & $0.060,4.149$ & 0.519 & 0.77 & $0.084,7.095$ & 0.820 \\
\hline \multicolumn{10}{|l|}{ Marital } \\
\hline Single & $199(54.7)$ & $46(12.6)$ & $27(7.4)$ & 1 & & & & & \\
\hline In a relationship/married & $47(12.9)$ & $14(3.8)$ & $10(2.7)$ & 1.15 & $0.516,2.548$ & 0.737 & 1.20 & $0.457,3.086$ & 0.724 \\
\hline Divorced/separated/widowed & $15(4.1)$ & $3(0.8)$ & $3(0.8)$ & 0.39 & $0.076,2.027$ & 0.264 & 0.76 & $0.165,3.532$ & 0.731 \\
\hline \multicolumn{10}{|l|}{ Education } \\
\hline Secondary and below & $71(19.5)$ & $17(4.7)$ & $14(3.8)$ & 1 & & & & & \\
\hline Pre-tertiary & $71(19.5)$ & $20(5.5)$ & $14(3.8)$ & 1.79 & $0.788,4.057$ & 0.164 & 1.73 & $0.669,4.468$ & 0.259 \\
\hline Tertiary and above & $119(32.7)$ & $26(7.1)$ & $12(3.3)$ & 1.70 & $0.770,3.769$ & 0.188 & 1.11 & $0.423,2.903$ & 0.835 \\
\hline \multicolumn{10}{|l|}{ Psychiatric diagnoses } \\
\hline Schizophrenia & $155(42.7)$ & $47(12.9)$ & $29(8)$ & 1 & & & & & \\
\hline Other non-affective psychotic disorders & $85(23.4)$ & $15(4.1)$ & $9(2.5)$ & 0.80 & $0.399,1.603$ & 0.528 & 1.01 & $0.421,2.437$ & 0.977 \\
\hline Affective psychotic disorders & $20(5.5)$ & $1(0.3)$ & $2(0.6)$ & 0.29 & $0.034,2.389$ & 0.248 & 1.40 & $0.230,8.502$ & 0.716 \\
\hline
\end{tabular}

${ }^{\wedge}$ Outcome variables are none, one comorbid, two or more comorbid conditions; OR is odds ratio; $95 \% \mathrm{CI}$ is $95 \%$ confidence intervals; values in bold indicate significance at $p<.05$

Table 4 Multiple linear regression analyses of comorbidity, DASS-21, and WHOQOL-BREF scores

\begin{tabular}{|c|c|c|c|c|c|c|c|c|}
\hline \multirow[t]{3}{*}{ Chronic conditions } & \multicolumn{8}{|c|}{ Quality of life (WHOQOL-BREF) } \\
\hline & \multicolumn{2}{|c|}{ Physical Health } & \multicolumn{2}{|c|}{ Psychological Health } & \multicolumn{2}{|c|}{ Social Relationships } & \multicolumn{2}{|c|}{ Environment } \\
\hline & $B(S E)$ & $95 \% \mathrm{CI}$ & $B(S E)$ & $95 \% \mathrm{CI}$ & $B(S E)$ & $95 \% \mathrm{CI}$ & $B(S E)$ & $95 \% \mathrm{CI}$ \\
\hline One or more & $-0.7(0.3)$ & $(-1.33-0.02)$ & $-0.3(0.4)$ & $(-1.05,0.51)$ & $-0.8(0.4)$ & $(-1.59,0.05)$ & $0.1(0.3)$ & $(-0.61,0.80)$ \\
\hline One & $-0.9(0.4)$ & $(-1.62-0.09)$ & $-0.6(0.5)$ & $(-1.46,0.34)$ & $-1.0(0.5)$ & $(-1.93,-0.03)$ & $-0.2(0.4)$ & $(-0.44,1.56)$ \\
\hline Two or more & $-0.4(0.5)$ & $(-1.30,0.54)$ & $0.2(0.6)$ & $(-0.90,1.32)$ & $-0.4(0.6)$ & $(-1.57,0.76)$ & $0.6(0.5)$ & $(-1.00,0.64)$ \\
\hline vs. None & Ref. & & Ref. & & Ref. & & Ref. & Ref. \\
\hline \multirow[t]{3}{*}{ Chronic conditions } & \multicolumn{8}{|c|}{ Psychological outcome measures (DASS 21) } \\
\hline & \multicolumn{3}{|c|}{ Anxiety } & \multicolumn{3}{|l|}{ Depression } & \multicolumn{2}{|c|}{ Stress } \\
\hline & \multicolumn{3}{|c|}{$B(S E)$} & $B(S E)$ & \multicolumn{2}{|l|}{$95 \% \mathrm{CI}$} & $B(S E)$ & $95 \%$ CI \\
\hline One or more & \multicolumn{2}{|c|}{$0.4(1.1)$} & $(-1.742 .60)$ & $1.6(1.3)$ & \multicolumn{2}{|c|}{$(-0.96,4.15)$} & $1.7(1.2)$ & $(-0.79,4.17)$ \\
\hline One & \multicolumn{2}{|c|}{$0.1(1.3)$} & $(-2.472 .57)$ & $2.0(1.5)$ & \multicolumn{2}{|c|}{$(-0.95,4.99)$} & $1.9(1.5)$ & $(-1.01,4.73)$ \\
\hline Two or more & \multirow{2}{*}{\multicolumn{2}{|c|}{1.1 (1.6) }} & $04.16)$ & $0.9(1.8)$ & \multicolumn{2}{|c|}{$(-2.76,4.50)$} & $1.4(1.8)$ & $(-2.124 .91)$ \\
\hline vs. None & \multicolumn{2}{|l|}{ Ref. } & & Ref. & & & Ref. & \\
\hline
\end{tabular}

Analyses involved regressing quality of life domains or psychological outcomes on the number of comorbid conditions (one or more vs. none; one, two or more, vs. none) while controlling for sociodemographic information such as gender, ethnicity, marital status, highest education levels, age, age of onset, and psychotic disorder categories; values in bold indicate significance at $p<.05$ 
may be positively linked to co-occurring physical health complications [48, 49]. However, due to the small numbers included in the affective psychotic disorder group, results pertaining to this group should be interpreted with caution. A recent report had found that cardiovascular, neurological, or skin-related diseases, each combined with respiratory diseases, had the highest impact on mortality rates [50]. As respiratory and skin conditions were reported in a sizable proportion of individuals, and as respiratory and skin conditions were significantly correlated locally, our findings, together with other evidences found overseas, would warrant a thorough examination of local data of these combinations in psychotic disorders.

Subsequent multinomial regression analyses revealed that the psychotic disorder type was not significantly associated with comorbidity after controlling for covariates, suggesting that other risk factors (i.e., age, age of onset, Malay and Indian ethnicities) played a more significant role. Age has been consistently documented to have a significant and important impact on comorbidity [15, 25, 26]. According to biomarker research, aging is accelerated in individuals with schizophrenia due to oxidative stress and inflammation, contributed by an interplay of unhealthy lifestyle habits and physiological abnormalities [51]. Moreover, a significant effect of an early age of onset could indicate that the vulnerabilities associated with those previously described physiopathological links, such as changes to immune system activity or unhealthy lifestyle behaviors found in individuals with schizophrenia, could be present at a young age thus making comorbidity more likely with age [52, 53].

Malay and Indian ethnic groups were at least three times more likely to have comorbid conditions. Our findings were expected as past research had shown greater prevalence of self-reported chronic conditions, such as diabetes, high cholesterol, and heart diseases, stroke, in Malay and/or Indian ethnic groups [21, 23, 54]. Various cardiovascular related complications, such as central arterial stiffness [55] and glycaemic control [56], have been found to be associated with Malay and/or Indian ethnic groups diagnosed with diabetes for instance, suggesting that lifestyle risk factors, such as diet, could have also contributed to the increased likelihood of certain comorbid presentations in these ethnic groups [22].

Being an ethnic minority could potentially compound the odds of receiving worse health outcomes over and above the aforementioned heightened risk of morbidity and mortality associated with comorbidity. This is due to known ethnic disparities associated with a myriad of factors, such as the association with social economic status (SES) [57]. Ethnic minorities could face increased barriers to primary care, experience lower health service engagement, all potentially leading to poorer health outcomes [58]. While our results imply a need for more awareness and sensitivity over these presentations among Malay and Indian adult patients with psychotic disorders, addressing financial concerns of low SES, could further uplift disparities and improve barriers to healthcare and health outcomes [57].

Adult individuals presenting with at least one co-occurring ICD-10 medical condition were more likely to self-rate higher anxiety, depression, stress levels, and lower physical health QOL than those without these presentations. When considering comorbid conditions only however, only physical health QOL tended to be negatively self-rated but not the psychological outcomes measured by DASS-21, which corroborated baseline findings from a past report [27]. In addition, when compared to those without comorbidity, individuals with one comorbid condition but not two or more comorbid conditions, were significantly associated with lower social relationships QOL and physical health QOL. To ascertain the reasons, additional post hoc descriptive and chi-square analyses were conducted. As compared to individuals with two or more comorbid conditions who had significantly greater proportions of those with comorbid respiratory, hypertension, diabetes/hyperglycemia, or hyperlipidemia conditions, individuals with one comorbid condition only had greater proportions of those with comorbid chronic pain (one vs two or more: $18.4 \%$ vs $7.8 \%$ with chronic pain conditions, though not significant). Thus, the association between physical health QOL and one comorbid condition only could be explained by a greater presence of comorbid chronic pain. The inverse relationship that was significant between having one comorbid condition only and social relationships domain of QOL could be explained by the fact that pain symptoms had been found to be temporally associated with a lack of a supportive social network $[59,60]$.

Similarly, post hoc descriptive analyses were conducted to explain the associations between QOL and ICD-10 medical conditions. Relative to those with one medical condition, individuals with two or more medical conditions who had significantly greater proportions of most conditions such as disorders of the circulatory system, nervous system, musculoskeletal, and digestive systems, could have experienced a lowered capacity to perform daily functions (i.e., a lack of energy, or needing medication to daily life functions) and therefore reported lower physical health QOL.

As a whole, the results gathered could be clarified by the fact that different medical conditions impact QOL and psychological health in many different ways. Since we analyzed a summation of disease indicators as opposed to single indicators (as the former is more parsimonious), it had inevitably created limits in the ways QOL or psychological outcomes could be analyzed. An additional limitation is that all medical conditions were treated as equally severe and the analyses were carried out without introducing weights to discern conditions by severity. Therefore, the results of our study are only preliminary and future research could expand the understanding of these relationships by addressing the 
severity and impacts of each comorbid condition on QOL or psychological outcomes. While this study did not take into account the severity of psychotic symptoms, it is expected that those who experience psychotic symptoms will self-rate higher psychological distress and lower QOL.

Other caveats concerning sample characteristics include the fact that the majority of participants had tertiary education. Thus, our study sample may not be sufficiently representative of the population of adults experiencing psychotic disorders due to possible selection bias.

To our knowledge, this study is among the few to investigate physical comorbidity in a large diverse sample of outpatients with psychotic disorders in a tertiary psychiatric care institution in a multi-ethnic non-western setting. Approximately three of ten individuals had at least one comorbid condition. While having at least one comorbid condition was significantly associated with lower quality of life in terms of physical health, the patterns of association between the different domains of QOL and comorbidity may vary depending on the types of medical condition. The majority of those with psychotic disorders reported co-morbid cardiovascular-related diseases. Skin and respiratory conditions were also found to co-occur in a sizable proportion of these individuals, warranting further research in this area. It was also found that individuals with schizophrenia had a significantly greater number of comorbid conditions as compared to other non-affective and affective psychotic disorders. However, additional analyses revealed that psychotic disorder type was not a significant predictor of comorbidity. Instead, older age, younger age of onset of psychotic disorders, Malay and Indian ethnic groups were stronger risk factors. Due to the nature of the study, the results of the study are preliminary and require further research to confirm the results.

Supplementary file2 (DOCX 16 KB)Supplementary Information The online version contains supplementary material available at https:// doi.org/10.1007/s00127-021-02034-8.

Funding This study was funded by National Medical Research Council (NMRC) under Centre Grant Seed Funding.

\section{Compliance with ethical standards}

Conflict of interest The authors declare that they have no conflicts of interest.

Open Access This article is licensed under a Creative Commons Attribution 4.0 International License, which permits use, sharing, adaptation, distribution and reproduction in any medium or format, as long as you give appropriate credit to the original author(s) and the source, provide a link to the Creative Commons licence, and indicate if changes were made. The images or other third party material in this article are included in the article's Creative Commons licence, unless indicated otherwise in a credit line to the material. If material is not included in the article's Creative Commons licence and your intended use is not permitted by statutory regulation or exceeds the permitted use, you will need to obtain permission directly from the copyright holder. To view a copy of this licence, visit http://creativecommons.org/licenses/by/4.0/.

\section{References}

1. Parks J, Svendsen D, Singer P, Foti ME, Mauer B (2006) Morbidity and mortality in people with serious mental illness. Alexandria VA Natl Assoc State Mental Health Prog Directors Med Directors Counc 25(4): 1-87

2. Lawrence D, Hancock KJ, Kisely S (2013) The gap in life expectancy from preventable physical illness in psychiatric patients in Western Australia: retrospective analysis of population based registers. BMJ 346:f2539

3. Stubbs B, Koyanagi A, Veronese N, Vancampfort D, Solmi M, Gaughran F et al (2016) Physical multimorbidity and psychosis: comprehensive cross sectional analysis including 242,952 people across 48 low-and middle-income countries. BMC Med 14(1):189

4. Lambert TJ, Velakoulis D, Pantelis C (2003) Medical comorbidity in schizophrenia. Med J Aust 178(9):S67

5. Brown S (1997) Excess mortality of schizophrenia: a meta-analysis. Br J Psychiatry 171(6):502-508

6. Laursen TM, Munk-Olsen T, Gasse C (2011) Chronic somatic comorbidity and excess mortality due to natural causes in persons with schizophrenia or bipolar affective disorder. PLoS ONE 6(9):e24597

7. van den Akker M, Buntinx F, Knottnerus JA (1996) Comorbidity or multimorbidity: what's in a name? A review of literature. Eur J Gener Pract 2(2):65-70

8. Feinstein AR (1970) The pre-therapeutic classification of comorbidity in chronic disease. J Chronic Dis 23(7):455-468

9. Oreški I, Jakovljević M, Aukst-Margetić B, Crnčević Orlić Ž, Vuksan-Ćusa BJPD (2012) Comorbidity and multimorbidity in patients with schizophrenia and bipolar disorder: similarities and differencies. Psychiatr Danub 24(1):80-85

10. Jones DR, Macias C, Barreira PJ, Fisher WH, Hargreaves WA, Harding CM (2004) Prevalence, severity, and co-occurrence of chronic physical health problems of persons with serious mental illness. Psychiatr Serv 55(11):1250-1257

11. Schoepf D, Uppal H, Potluri R, Heun R (2014) Physical comorbidity and its relevance on mortality in schizophrenia: a naturalistic 12-year follow-up in general hospital admissions. Eur Arch Psychiatry Clin Neurosci 264(1):3-28

12. Leucht S, Burkard T, Henderson J, Maj M, Sartorius N (2007) Physical illness and schizophrenia: a review of the literature. Acta Psychiatr Scand 116(5):317-333

13. Carney CP, Jones L, Woolson RF (2006) Medical comorbidity in women and men with schizophrenia. J Gen Intern Med 21(11):1133-1137

14. Morgan VA, Waterreus A, Jablensky A, Mackinnon A, McGrath JJ, Carr V et al (2012) People living with psychotic illness in 2010: the second Australian national survey of psychosis. Aust N Z J Psychiatry 46(8):735-752

15. Marengoni A, Angleman S, Melis R, Mangialasche F, Karp A, Garmen A et al (2011) Aging with multimorbidity: a systematic review of the literature. Ageing Res Rev 10(4):430-439

16. Smith DJ, Langan J, McLean G, Guthrie B, Mercer SW (2013) Schizophrenia is associated with excess multiple physical-health comorbidities but low levels of recorded cardiovascular disease in primary care: cross-sectional study. BMJ Open 3(4):e002808 
17. Zimmet PZ, Magliano DJ, Herman WH, Shaw JE (2014) Diabetes: a 21st century challenge. Lancet Diabetes Endocrinol 2(1):56-64

18. Bailey S, Gerada C, Lester H, Shiers D (2012) The cardiovascular health of young people with severe mental illness: addressing an epidemic within an epidemic. Psychiatrist 36(10):375-378

19. World Health Organization (2012) Noncommunicable diseases in the South-East Asia Region, 2011: situation and response

20. Palaniappan L, Wang Y, Fortmann SP (2004) Coronary heart disease mortality for six ethnic groups in California, 1990-2000. Ann Epidemiol 14(7):499-506

21. National Health Survey 2010 (2011) In M. O. H. Singapore (Ed.).

22. Health Promotion Board (2010) National Nutrition Survey 2010. In R. S. P. Division (Ed.).

23. Teh WL, Abdin E, Vaingankar JA, Seow E, Sagayadevan V, Shafie $S$ et al (2018) Prevalence of stroke, risk factors, disability and care needs in older adults in Singapore: results from the WiSE study. BMJ Open 8(3):e020285

24. Subramaniam M, Abdin E, Vaingankar J, Shafie S, Chua B, Sambasivam $\mathrm{R}$ et al (2020) Tracking the mental health of a nation: prevalence and correlates of mental disorders in the second Singapore mental health study. Epidemiol Psychiatr Sci 29:1-10

25. Chong SA, Abdin E, Nan L, Vaingankar JA, Subramaniam M (2017) Prevalence and impact of mental and physical comorbidity in the adult Singapore population. Ann Acad Med Singap 41(3): 105

26. Subramaniam M, Abdin E, Picco L, Vaingankar JA, Chong SA (2014) Multiple chronic medical conditions: prevalence and risk factors-results from the Singapore Mental Health Study. Gen Hosp Psychiatry 36(4):375-381

27. Sim K, Chan YH, Chua TH, Mahendran R, Chong SA, McGorry P (2006) Physical comorbidity, insight, quality of life and global functioning in first episode schizophrenia: a 24-month, longitudinal outcome study. Schizophr Res 88(1-3):82-89

28. Lim MW, Lee J (2018) Determinants of health-related quality of life in schizophrenia: beyond the medical model. Front Psychiatry 9:712

29. Grimby A, Svanborg A (1997) Morbidity and health-related quality of life among ambulant elderly citizens. Aging Clin Exp Res 9(5):356-364

30. Subramaniam M, Abdin E, Vaingankar JA, Nan L, Heng D, McCrone $P$ et al (2013) Impact of psychiatric disorders and chronic physical conditions on health-related quality of life: Singapore Mental Health Study. J Affect Disord 147(1-3):325-330

31. American Psychological Association (2000) Diagnostic and statistical manual of mental disorders 4th ed., text revision. Washington: DC

32. Caton CL, Drake RE, Hasin DS, Dominguez B, Shrout PE, Samet $S$ et al (2005) Differences between early-phase primary psychotic disorders with concurrent substance use and substance-induced psychoses. Arch Gen Psychiatry 62(2):137-145

33. Lovibond PF, Lovibond SH (1995) The structure of negative emotional states: Comparison of the Depression Anxiety Stress Scales (DASS) with the beck depression and anxiety inventories. Behav Res Ther 33(3):335-343

34. Antony MM, Bieling PJ, Cox BJ, Enns MW, Swinson RP (1998) Psychometric properties of the 42-item and 21-item versions of the Depression Anxiety Stress Scales in clinical groups and a community sample. Psychol Assess 10(2):176

35. WHOQOL-BREF Introduction, Administration, Scoring and Generic Version of the Assessment (1996) Field Trial Version. pp. $1-18$

36. Trompenaars FJ, Masthoff ED, Van Heck GL, Hodiamont PP, De Vries J (2005) Content validity, construct validity, and reliability of the WHOQOL-Bref in a population of Dutch adult psychiatric outpatients. Qual Life Res 14(1):151-160

37. Ferraro KF, Wilmoth JM (2000) Measuring morbidity: disease counts, binary variables, and statistical power. J Gerontol Ser B Psychol Sci Soc Sci 55(3):S173-S189
38. Subramaniam M, Vaingankar J, Heng D, Kwok K, Lim Y, Yap M et al (2012) The Singapore Mental Health Study: an overview of the methodology. Int J Methods Psychiatr Res 21(2):149-157

39. Batki SL, Meszaros ZS, Strutynski K, Dimmock JA, Leontieva L, Ploutz-Snyder R et al (2009) Medical comorbidity in patients with schizophrenia and alcohol dependence. Schizophr Res 107(2-3):139-146

40. Subramaniam M, Chong S-A, Pek E (2003) Diabetes mellitus and impaired glucose tolerance in patients with schizophrenia. Can J Psychiatry 48(5):345-347

41. Hung C-F, Wu C-K, Lin P-Y (2005) Diabetes mellitus in patients with schizophrenia in Taiwan. Progress Neuro-Psychopharmacol Biol Psychiatry 29(4):523-527

42. Ferentinos P, Dikeos D (2012) Genetic correlates of medical comorbidity associated with schizophrenia and treatment with antipsychotics. Curr Opin Psychiatry 25(5):381-390

43. Fernandez-Egea E, Bernardo M, Donner T, Conget I, Parellada E, Justicia A et al (2009) Metabolic profile of antipsychoticnaive individuals with non-affective psychosis. Br J Psychiatry 194(5):434-438

44. Fernandez-Egea E, Bernardo M, Heaphy CM, Griffith JK, Parellada E, Esmatjes E et al (2009) Telomere length and pulse pressure in newly diagnosed, antipsychotic-naive patients with nonaffective psychosis. Schizophr Bull 35(2):437-442

45. Manzanares N, Monseny R, Ortega L, Montalvo I, Franch J, Gutiérrez-Zotes A et al (2014) Unhealthy lifestyle in early psychoses: the role of life stress and the hypothalamic-pituitary-adrenal axis. Psychoneuroendocrinology 39:1-10

46. Burti L, Berti L, Bonfioli E, Fiorini I (2013) Meeting the challenge of physical comorbidity and unhealthy lifestyles. Improv Mental Health Care Glob Chall 3:114-130

47. Möller HJ, Bottlender R, Wegner U, Wittmann J, Strauß A (2000) Long-term course of schizophrenic, affective and schizoaffective psychosis: focus on negative symptoms and their impact on global indicators of outcome. Acta Psychiatr Scand 102:54-57

48. Nuevo R, Chatterji S, Fraguas D, Verdes E, Naidoo N, Arango C et al (2011) Increased risk of diabetes mellitus among persons with psychotic symptoms: results from the WHO World Health Survey. J Clin Psychiatry 72(12):1592-1599

49. Moreno C, Nuevo R, Chatterji S, Verdes E, Arango C, Ayuso-Mateos JL (2013) Psychotic symptoms are associated with physical health problems independently of a mental disorder diagnosis: results from the WHO World Health Survey. World Psychiatry 12(3):251-257

50. Kugathasan $P, W u ~ H$, Gaughran F, Nielsen RE, Pritchard M, Dobson $R$ et al (2020) Association of physical health multimorbidity with mortality in people with schizophrenia spectrum disorders: Using a novel semantic search system that captures physical diseases in electronic patient records. Schizophr Res 216:408-415

51. Nguyen TT, Eyler LT, Jeste DV (2017) Systemic biomarkers of accelerated aging in schizophrenia: a critical review and future directions. Schizophr Bull 44(2):398-408

52. Rubinow KB, Rubinow DR (2017) In immune defense: redefining the role of the immune system in chronic disease. Dialogues Clin Neurosci 19(1):19

53. Pillinger T, Beck K, Gobjila C, Donocik JG, Jauhar S, Howes OD (2017) Impaired glucose homeostasis in first-episode schizophrenia: a systematic review and meta-analysis. JAMA Psychiatry 74(3):261-269

54. Hong CY, Chia KS, Hughes K, Ling SL (2004) Ethnic differences among Chinese, Malay and Indian patients with type 2 diabetes mellitus in Singapore. Singap Med J 45(4):154-160

55. Zhang X, Liu JJ, Sum CF, Ying YL, Tavintharan S, Ng XW et al (2015) Ethnic disparity in central arterial stiffness and its determinants among Asians with type 2 diabetes. Atherosclerosis 242(1):22-28 
56. Ng TP, Goh LG, Tan Y, Tan E, Leong H, Tay EG et al (2005) Ethnic differences in glycaemic control in adult Type 2 diabetic patients in primary care: a 3-year follow-up study. Diabet Med 22(11):1598-1604

57. Cook BL, Trinh N-H, Li Z, Hou SS-Y, Progovac AM (2017) Trends in racial-ethnic disparities in access to mental health care, 2004 2012. Psychiatr Serv 68(1):9-16

58. Anderson NB, Bulatao RA, Cohen B, on Race P, National Research C (2004) Race/ethnicity, socioeconomic status, and health. In:
Critical perspectives on racial and ethnic differences in health in late life. National Academies Press, US

59. Karayannis NV, Baumann I, Sturgeon JA, Melloh M, Mackey SC (2018) The impact of social isolation on pain interference: a longitudinal study. Ann Behav Med 53(1):65-74

60. Hughes S, Jaremka LM, Alfano CM, Glaser R, Povoski SP, Lipari AM et al (2014) Social support predicts inflammation, pain, and depressive symptoms: Longitudinal relationships among breast cancer survivors. Psychoneuroendocrinology 42:38-44 